\title{
Infection au nouveau coronavirus chez un professionnel de la santé suite à un accès palustre
}

\section{Infection with the new coronavirus in a health professional following an attack of malaria}

\author{
Mama Cisse $\mathrm{I}^{1}$, Alassani $\mathrm{A}^{1}$, Adjobimey $\mathrm{M}^{2}$, Mikponhoue $\mathrm{R}^{2}$, Hinsou $\mathrm{Av}^{2}$, Attinsounon $\mathrm{A}^{1}$, \\ Gandaho $\mathrm{P}^{1}$ \\ 1-Département de Médecine et Spécialités Médicales, Faculté de Médecine, Université de Parakou, Bénin \\ 2-Département de Médecine et Spécialités Médicales, Faculté des Sciences de la Santé Médecine, \\ Université d'Abomey Calavi, Bénin
}

Auteur correspondant : Alassani Adébayo, Médecin interniste, Assistant Chef de Clinique, Faculté de Médecine, Université de Parakou, Mail: debayoalassani@gmail.com

\begin{abstract}
Résumé
Introduction : la COVID-19 constitue une pandémie. Le personnel de santé constitue un groupe à risque. L'objectif de travail était de rapporter un cas clinique d'infection au nouveau coronavirus chez un personnel soignant.

Observation : il s'est agi une infection au COVID-19 diagnostiquée chez une jeune femme, agent de santé, âgée de 31 ans et qui ne présentait aucun antécédent médical suite à l'évolution défavorable d'un accès palustre simple. Les manifestations présentées par la patiente étaient dominées par la fièvre, l'asthénie, les céphalées et la toux. Le diagnostic a été posé par la RT-PCR alors que le TDR était négatif. L'évolution a été favorable sous chloroquine, zinc et azithromycine.
\end{abstract}

Conclusion : Le dépistage de la COVID-19 doit être systématique devant l'évolution sous traitement défavorable du paludisme.

Mots-clés : COVID-19, Personnel de santé, Bénin

\begin{abstract} chloroquine, zinc and azithromycin.

Keywords: COVID-19, Health personnel, Benin.

\section{Introduction}

L'infection au nouveau coronavirus constitue un problème de santé publique. Il s'agit d'une infection qui concerne tous les pays au monde et a été déclarée comme pandémie depuis le 11 mars 2020 [1]. A la date du 13 juillet 2020, plus de 12 millions de personnes atteintes et 556075 de décès. L'Afrique relativement moins atteinte mais enregistre des milliers de cas et des centaines de décès. La période d'incubation de la COVID-19 est de 1 à 14 jours et souvent responsable d'une infection respiratoire simple avec fièvre et toux pendant 2 à 3 jours mais peut
\end{abstract}

Introduction: COVID-19 constitutes a pandemic. Health personnel are a risk group. The objective of the work was to report a clinical case of infection with the novel coronavirus in healthcare workers.

Observation: this was a COVID-19 infection diagnosed in a young woman, a health worker, age 31 years and who had no medical history following the unfavorable course of simple malaria access. The manifestations presented by the patient were dominated by fever, asthenia, headache and cough. The diagnosis was made by RT-PCR when the RDT was negative. The outcome was favorable with

Conclusion: Screening for COVID-19 should be systematic in the face of malaria treatment failure.

être grave chez certaines personnes avec une mortalité importante [2]. Les facteurs de risque de développer la maladie et de décès sont l'âge avancé, le diabète, les maladies cardiovasculaires et respiratoires [3]. Dans l'étude de Wu et al. [4], la prévalence du COVID-19 dans la population générale est de $2,3 \%$ tandis que chez les sujets atteints de maladies cardiovasculaires elle est de $10,5 \%$, chez les diabétiques $7,3 \%$, chez les sujets ayant une insuffisance respiratoires chroniques $6,3 \%$, chez les sujets atteints de cancer $5,6 \%$. Les principales voies de contamination de la COVID-19 sont 
celle aérienne et au contact des sécrétions digestives; la transmission verticale reste encore à prouver même si la transmission de plusieurs infections notamment virales a été observée [5]. Le personnel soignant étant en contact avec les sujets atteints est particulièrement à risque et peut à son tour contaminer les patients constituant ainsi un cercle vicieux favorisant la propagation et la pérennisation de la maladie. Durant l'épidémie de SARS-CoV-1en 2003, 21\% des sujets infectés sont le personnel soignant [6]. En janvier 2020, le personnel soignant constituait $29 \%$ des personnes atteintes de COVID-19 à Wuhan [7]. Au Bénin, à la date du 17 juillet 2020, 1602 cas de COVID-19 ont été confirmés dont et 31 sont décédés. Peu de données sur l'infection au nouveau coronavirus chez les agents de santé existent. La présente étude révèle un cas de COVID-19 chez un agent de santé qui est survenue dans un contexte particulier d'échec d'un traitement antipaludique.

\section{Observation}

Il s'agit d'une femme, âgée de 31 ans, célibataire, agent de santé en service à quelques dizaines de $\mathrm{km}$ de Parakou. Elle a été référée à Parakou pour une toux persistante dans un contexte d'altération de l'état général le $18 / 6 / 2020$. Le début de la symptomatologie remontait au 14/6/2020 avec une fièvre et des céphalées ayant motivé la réalisation d'un bilan où le diagnostic d'accès palustre simple biologiquement confirmé a été posé. Elle a bénéficié d'un traitement par artéméther $20 \mathrm{mg}$ et luméfantrine $120 ; 4$ comprimés matin et 4 comprimés soir débuté le même jour. La persistance des signes a motivé l'ajout au quatrième jour après le début du traitement antipalustre de ofloxacine $200 \mathrm{mg} 1$ comprimé matin et 1 comprimé soir. L'aggravation des signes associée à l'apparition de la toux, d'une agueusie, d'une anosmie, des myalgies et d'une asthénie motiva son transfert au Centre Hospitalier Universitaire du Borgou pour une meilleure prise en charge le 18/6/2020. Les antécédents personnels médicaux et chirurgicaux de cette patiente étaient sans particularité. Sur le plan gynéco-obstétrical, elle n'a pas eu de grossesse et n'avait jamais présenté d'allergie médicamenteuse; elle était porteuse de trait drépanocytaire AC. L'examen clinique notait: un état général altéré stade 3 OMS, des muqueuses palpébrales et bulbaires normocolorées, une température à $38,7^{\circ} \mathrm{C}$, un pouls à 96 par minute, une fréquence respiratoire à 28 cycles par minutes et une tension artérielle à 130/80 mm Hg. Elle présentait un bon état d'hydratation et une conscience normale.

L'examen pleuro-pulmonaire a noté un syndrome de condensation pulmonaire bilatérale. Le reste des appareils était normal. Sur le plan paraclinique on notait: un TDR (Test de Diagnostic Rapide) à la recherche des anticorps anti SARS-COV2 négatif le 18/6/2020 et une RT-PCR (Reverse Transcriptase Polymerase Chain Reaction) SARS-COV2 positive sur les deux prélèvements réalisés le 19/6/2020. Concernant les autres éléments du bilan: CRP négative, Hémogramme: anémie modérée $(11 \mathrm{~g} / \mathrm{dL})$ microcytaire (VGM 74$)$ hypochrome (TCMH 24,6), Créatinémie normale, Ionogramme sanguin: Hypokaliémie à 3,44 meq/L, Transaminases normales

L'imagerie n'a pas été réalisée

Le diagnostic retenu était celui pneumonie virale au Covid19

Traitement :

- Chloroquine $250 \mathrm{mg}$ : 1 comprimé matin, midi et soir

- Zinc $20 \mathrm{mg}$ : 1 comprimé le matin

- Vitamine $\mathrm{C} 1 \mathrm{~g}: 1$ comprimé à midi

- Azithromycine $250 \mathrm{mg}: 1$ comprimé le matin

- Paracétamol 500 mg : 2 comprimés matin, midi et soir

- Lanzoprazole $30 \mathrm{mg}$ : 1 gélule matin et soir

- Potassium $600 \mathrm{mg}$ : 1 gélule les matins

Evolution

-Aprexie à $\mathrm{J} 2$ d'hospitalisation

-Amendement de la toux, des myalgies et des céphalées à $\mathrm{J} 5 \mathrm{~d}$ 'hospitalisation

- A J13 d'hospitalisation, la patiente ne présentait aucune plainte; son examen clinique était normal, ce qui a motivé son exéat dans la soirée du $7 / 2020$.

\section{Discussion}

Le présent cas clinique décrit une infection au nouveau coronavirus chez un agent de santé. Le diagnostic de cette infection a été fait 5 jours après le début de la symptomatologie. Ce délai de diagnostic de la COVID-19 était proche de celui de Nishiura [8] au Japon qui variait entre 3 à 4 jours. La particularité de ce cas est sa survenue après un accès palustre biologiquement confirmé ce qui a favorisé le retard du diagnostic. Il s'agissait d'un sujet jeune sans antécédents médicaux ce qui contraste avec la 
littérature qui rapporte que la plupart des sujets atteints étaient des sujets âgés et ayant des pathologies chroniques comme le diabète, le cancer, les maladies cardiovasculaires et respiratoires [3]. Les arguments expliquant la COVID-19 dans ce cas peut s'expliquer par l'immunodépression passagère qu'engendrent les affections aiguës comme le paludisme favorisant la multiplication des agents pathogènes comme les virus. Il n'est pas rare d'observer des cas d'infection herpétique en cas d'infections aiguës qui devraient être considérées désormais comme étant des facteurs favorisants la survenue de la COVID-19. Dans une étude réalisée aux Etats Unis chez le personnel soignant, la moyenne d'âge des sujets atteints de la COVID-19 était 42 ans avec des extrêmes de 32 à 54 ans [9]; preuve que cette pathologie peut se rencontrer chez les sujets jeunes. Les principales manifestations de la COVID-19 rapportées dans la littérature étaient dominées par la fièvre, la toux, les céphalées et la dyspnée [10,11]. Dans l'étude de Forouzesh [12], l'hypogueusie et l'anosmie ont été également rapportées et considérées comme les manifestations neurologiques. Ces différents signes sont retrouvés dans le cas présent. Sur le plan biologique, une anémie légère avait été observée. Dans l'étude de Guan et al. [13], 53,6 $\%$ des patients atteints de la COVID-19 étaient anémiés. Le diagnostic de la COVID-19 a été fait par le TDR et la RT-PCR conformément aux recommandations internationales [14]. Ces deux techniques de diagnostic ne sont pas égales car le TDR a l'avantage d'être moins cher, rapidement réalisable mais a une faible sensibilité; de plus la production des anticorps est plus retardée rendant le diagnostic tardif de la COVID-19 [15]. Ceci explique la négativité du TDR et la positivité de la RT-PCR dans le présent cas. Le traitement avait fait appel entre autres à la chloroquine et l'azithromycine. Dans les études de Colson [16] et Liying [17], l'efficacité de la chloroquine pour le traitement de la COVID-19 a été prouvée. Les patients atteints de pneumopathie ont eu une diminution significative de la durée d'hospitalisation et de la mortalité lorsqu'ils ont été traités par la chloroquine dans l'étude de Gao et al [18]. Dans l'étude de Gautret [19], l'association chloroquine et azithromycine a montré une efficacité supérieure à la monothérapie à base de chloroquine ou d'azithromycine. La durée d'hospitalisation de 13 jours était conforme à la moyenne de 5 à 25 jours rapportée par Chen [20]. L'évolution a été favorable ceci du fait du jeune âge de la patiente et l'absence de comorbidité. Dans l'étude de Ji [21], l'âge moyen de décès était de 78 ans et seulement les sujets cumulant plusieurs comorbidités sont concernés. Seulement 2,8\% de décès n'avaient pas d'antécédents médicaux pathologiques en Italie [2]

\section{Conclusion}

La COVID-19 peut survenir après un paludisme. Son dépistage doit être systématique devant l'évolution défavorable malgré un traitement adéquat d'une pathologie diagnostiquée. Une attention particulière doit être accordée au personnel soignant en contact permanent avec les patients sains ou infectés.

Conflit d'intérêt : Aucun

Contribution des auteurs: MCI a initié le travail, $\mathbf{A A}$ a écrit le manuscrit, $\mathbf{A M}$ a réalisé les statistiques, MR, HAV, AP ont lu et corrigé le manuscrit

Remerciements : A l'endroit de tout le personnel soignant impliqué dans la prise en charge de la COVID-19.

\section{Références}

1-World Health Organization. WHO declares COVID-19 a pandemic. 2020. https://www.who.int/dg/speec hes/detai 1/whodirector-general-s-opening-remarks-at-the-media -briefing-on-covid-19-11-march -2020. Accessed July 17, 2020.

2-Yang X, Yu Y, Xu J, Shu H, Xia J, Liu H, et al. 2020. Clinical course and outcomes of critically ill patients with SARS-CoV-2 pneumonia in Wuhan, China: a single-centered, retrospective, observational study. Lancet Respir Med; 20: 30079-5.

3- Yang J, Zheng Y, Gou X, Pu K, Chen Z et al. 2020. Prevalence of comorbidities in the novel Wuhan coronavirus (COVID-19) infection: a systematic review and meta-analysis. International Journal of Infectious Diseases; 1(3): 1-15 doi: 10.1016/j.ijid.2020.03.017

4- Wu A, Peng Y, Huang B, Ding X, Wang X, Niu $P$, et al. 2020. Genome Composition and Divergence of the Novel Coronavirus (2019nCoV) Originating in China. Cell Host Microbe; 5(6) : 54-62

5-Bulut C, Kato Y. Epidemiology of COVID-19. 2020. Turkish Journal of Medical Sciences; 50: $563-70$

6- Johnston LB, Conly JM. 2004. Severe acute respiratory syndrome: What have we learned two years later? Can J Infect Dis Med Microbiol; 15(6): 309-12. 
7- Wang D, Hu B, Hu C. 2020. Clinical characteristics of 138 hospitalized patients with 2019 novel coronavirus-infected pneumonia in Wuhan, China. JAMA; 323(11):1061-9. 8-Nishiura H, Linton NM, Akhmetzhanov AR. 2020. Serial interval of novel coronavirus (COVID-19) infections. Int J Infect Dis;93: 2846.

9-US Department of Health and Human Services/Centers for Disease Control and Prevention. 2020. Characteristics of Health Care Personnel with COVID-19-United States, February 12-April 9, 2020. Morbidity and Mortality Weekly Report; 69(15) : 477-51

10-Huang C, Wang Y, Li X, Ren L, Zhao J, Hu $\mathrm{Y}$ et al. 2020. Clinical features of patients infected with 2019 novel coronavirus in Wuhan, China. Lancet; 395: 497-506.

11-Xu X, Han M, Li T, Sun W, Wang D, Fu B et al. 2020. Effective treatment of severe COVID19 patients with tocilizumab. PNAS; 117(20): 10970-5

12-Forouzesh M, Rahimi A, Valizadeh R, Dadashzadeh N, Mirzazadeh A. 2020. Clinical display, diagnostics and genetic implication of Novel Coronavirus (COVID-19). Epidemic. European Review for Medical and Pharmacological Sciences; 24: 4607-15

13-Guan W J, Ni Z Y, Hu Y. 2020. Clinical Characteristics of Coronavirus Disease 2019 in China. N Engl J Med. DOI: 10.1056/NEJMoa2002032

14-Islam H, Rahman A, Masud J, Shweta DS, Araf Y, Ullah MA et al. 2020. A Generalized Overview of SARS-CoV-2: Where Does the Current Knowledge Stand? Electron J Gen Med;17(6): 1-21

15- Loeffelholz MJ, Tang Y. 2020. Laboratory diagnosis of emerging human coronavirus infections - the state of the art, Emerging Microbes \& Infections; 9(1); 747-56
16-Colson P, Rolain JM, Lagier JC, Brouqui P, Raoult D. 2020. Chloroquine and hydroxychloroquine as available weapons to fight COVID-19, International Journal of Antimicrobial Agents, doi: https://doi.org/10.1016/j.ijantimicag.2020.10593 2

17-Liying D, Shasha H, Jianjun G. 2020. Discovering drugs to treat coronavirus disease 2019 (COVID-19). Drug Discoveries \& Therapeutics; 14(1):58-60

18-Gao J, Tian Z, Yang X. 2020. Breakthrough: Chloroquine phosphate has shown apparent efficacy in treatment of COVID-19 associated pneumonia in clinical studies. BioScience Trends;14(1). https://doi.org/10.5582/ bst.2020.01047 PMid:32074550

19-Gautret P, Lagier JC, Parola P. 2020. Hydroxychloroquine and azithromycin as a treatment of COVID-19: results of an open-label non-randomized clinical trial. Int $\mathrm{J}$ Antimicrob Agents DOI:10.1016/j.ijantimicag.2020.105949

20-Chen N, Zhou M, Dong X. 2020. Epidemiological and clinical characteristics of 99 cases of 2019 novel coronavirus pneumonia in Wuhan, China: a descriptive study. Lancet 395(10223): 507-13

21-Ji Y, Ma Z, Peppelenbosch MP, Pan Q. 2020.

Potential association between COVID-19 mortality and health-care resource availability. The Lancet Global Health; 8 (4): e480. doi: 10.1016/S2214-109X(20)30068-1

22-Istituto Superiore di Sanità. 2020. Characteristics of COVID-19 patients dying in Italy Report based on available data on April 2nd, $2020 \quad$ [online]. Website https://www.epicentro.iss.it/en/coronavirus/bollet tino/Report-COVID 2019_2_april_2020. pdf [accessed 17 July 2020]. 